Practice article

\title{
Robust track following in Hard Disk Drives with time delays: An infinite dimensional approach
}

\author{
Peng Yan ${ }^{\mathrm{a}, *}$, Hitay Özbay ${ }^{\mathrm{b}}$, Zeshan Lyu $^{\mathrm{c}}$ \\ a Key Laboratory of High-efficiency and Clean Mechanical Manufacture (Shandong University), Ministry of Education, School of Mechanical \\ Engineering, Shandong University, Jinan, Shandong, 250061, China \\ ${ }^{\mathrm{b}}$ Electrical and Electronics Engineering Department, Bilkent University, Ankara, 06800, Turkey \\ c School of Automation Science and Electrical Engineering, Beihang University, Beijing, 100191, China
}

\section{H I G H L I G H T S}

- The development of an infinite dimensional robust head positioning algorithm for HDD with time delays.

- Non-fragile digital implementation incorporating sampled-data FIR filter approximation.

- Significant improvements on tracking TPI capability and transient responses measurement.

\section{A R T I C L E I N F O}

\section{Article history:}

Received 14 August 2016

Received in revised form 30 December 2018

Accepted 18 March 2019

Available online 21 March 2019

\section{Keywords:}

Mechatronics

Servo

Hard Disk Drives

Robust control

Time delays

\begin{abstract}
A B S T R A C T
Due to the existence of various sources of delays, the dynamical model of HDD (Hard Disk Drive) servo systems is actually infinite dimensional, although most of the existing algorithms simplified the model with Padé expansions or other finite dimensional approximations. In this paper, a robust loop shaping algorithm is developed for high accuracy track following of HDD servo systems with delays by using an $\mathcal{H}^{\infty}$ synthesis approach for infinite dimensional systems. The $\mathcal{H}^{\infty}$ controller is derived with a structure of an internal feedback loop including an FIR (Finite Impulse Response) filter and a first order IIR (Infinite Impulse Response) filter, which facilitates stable implementations. Digital implementation of the proposed control structure is further investigated by incorporating $\mathcal{H}^{\infty}$ based sampled-data FIR filter design and approximation. Comparisons to other robust control methods are given, and the advantages of the proposed algorithm are demonstrated in terms of tracking TPI (Track-per-Inch) capability and transient responses using HDD factory measurement data.
\end{abstract}

(c) 2019 ISA. Published by Elsevier Ltd. All rights reserved.

\section{Introduction}

The rapid growth of research on nano-precision mechatronics and the immense demands of HDD storage with various applications have imposed many challenges on servo technologies. There have been considerable research works that address important control issues inherited in this area. We would like to refer to [16] for tutorials on modern HDD servo systems. According to the operations of HDD servo systems supporting data read/write access, there are mainly three modes for servo control: track following, track seeking, and seek settling. In track following mode, the head is positioned within the track OCLim (Off-CenterLimit), where the servo control algorithms are responsible for attenuating all possible periodic disturbances (Repeatable Runout) and random disturbances (Non-repeatable Runout) to achieve minimized TMR (track misregistration). Track seeking and seek

\footnotetext{
* Corresponding author.

E-mail address: pengyan2007@gmail.com (P. Yan).
}

settling modes are responsible for trajectory control of head motion from one track to another, and the transition from track seeking to track following, respectively.

Today's high end HDDs can achieve more than $300 k$ TPI (Trackper-Inch), which corresponds to the track width of less than $100 \mathrm{~nm}$. Unlike other nano-positioning systems, HDD servo systems have to be very cost-effective and robust due to the commercial requirements, which poses many challenges from servo control perspectives, and have attracted significant research efforts, such as runout compensations [7-10], track seeking control [11-13], servo-pattern writing algorithms in HDD manufacturing $[14,15]$, as well as robust vibration control with a selfsensing mechanism [16].

Among the abundant research results on HDD servo algorithms, one of the central topics is the optimal robust loop shaping problem for HDD track following, which determines the servo bandwidth, attenuations of disturbance, and transient behaviors of head motions. Robust control theory has been widely investigated for HDD servo loop shaping [17,18], as well as other 
nano-positioning applications [19-21], where standard $\mathcal{H}^{\infty}$ control [17] and $\mu$-synthesis [18] algorithms have been successfully developed. The problem is further explored in more recent publications. In [22] a user-friendly loop-shape optimization method was developed for HDD head-positioning by employing the Robust Bode (RBode) plot. Synthesis and implementation of fractional order controllers were discussed for HDD servo systems in [23]. Meanwhile optimal $\mathcal{H}^{\infty}$ control structure was investigated in [24] for periodically time-varying systems to handle servo-sample missing and periodical disturbances in HDD control. A very recent result in [25] indicates that data-based tuning of disturbance observer can be employed for the enhancement of sensitivity loop shaping.

It is worth noting that the model of HDD servo systems is actually infinite dimensional due to the existence of various sources of delays (see [6] for details of industry examples). Available approaches such as [26] and [27] are very complicated and limited to specific models (e.g. first order systems). For application purposes, most existing results simplify the model with Padé expansion or other finite dimensional approximations. Therefore two important questions remain open in this area: (1) is it possible to synthesize robust $\mathcal{H}^{\infty}$ controllers for the infinite dimensional HDD model directly, and (2) what are the advantages of the direct approach, compared to the existing approximation methods?

In this paper, the time delay model of HDD servo systems is analyzed with uncertainties and formulated to a mixed sensitivity optimization problem for robust control. By using the $\mathcal{H}^{\infty}$ control theory for infinite dimensional systems [28], the optimal $\mathcal{H}^{\infty}$ performance can be numerically computed and an explicit form of the optimal $\mathcal{H}^{\infty}$ controller can be developed. Based on coprimeinner/outer factorization, we can eliminate the unstable pole-zero cancelations in the $\mathcal{H}^{\infty}$ controller and generate the controller structure with an internal feedback loop including an FIR (Finite Impulse Response) filter and an IIR (Infinite Impulse Response) filter [29], which facilitates a non-fragile implementation of the robust servo controller. Inspired by [30,31], digital implementation of the proposed control structure is further investigated by incorporating $\mathcal{H}^{\infty}$ based sampled-data FIR filter design and approximation, where the optimal coefficients of the sampled-data FIR filter can be derived by an LMI-based algorithm. Comparisons with existing robust control methods demonstrate significant improvements of tracking TPI (Track-per-Inch) capability and transient responses for seek settling.

The rest of the paper is organized as follows. Some preliminaries on infinite dimensional $\mathcal{H}^{\infty}$ control are first given in Section 2. In Section 3, we describe the HDD servo model with time delays and uncertainties. In Section 4, we discuss the mixed sensitivity optimization problem for the SISO time delay systems, and the non-fragile structure for controller implementations, as well as $\mathcal{H}^{\infty}$ based sampled-data FIR filter design and approximations. An industrial design case is studied in details in 5, where comparisons are also provided with existing robust control approaches of Padé approximations. Tracking TPI capabilities and TMR analysis are discussed in Section 6 to illustrate the advantages of the proposed design, followed by conclusions in Section 7.

\section{Preliminaries}

We first recall some results on the following two-block $\mathcal{H}^{\infty}$ optimization problem

$\gamma_{0}:=\inf _{C \text { stab. } P_{0}}\left\|\left[\begin{array}{l}W_{1} S \\ W_{2} T\end{array}\right]\right\|_{\infty}$,

where $S=\left(1+P_{0} C\right)^{-1}$ and $T=P_{0} C\left(1+P_{0} C\right)^{-1}$ are the sensitivity and complementary sensitivity functions of the plant model
$P_{0}(s)$, and $W_{1}, W_{2}$ are rational weighting functions which satisfy $\left(W_{2} N_{o}\right),\left(W_{2} N_{o}\right)^{-1} \in \mathcal{H}^{\infty}\left(\mathbb{C}_{+}\right)$. Note that the plant model $P_{0}(s)$ considered here can be infinite dimensional, with the coprime factorization in the form

$P_{0}(s)=\frac{m_{n}(s) N_{o}(s)}{m_{d}(s)}$,

where $m_{n} \in \mathcal{H}^{\infty}\left(\mathbb{C}_{+}\right)$is inner (all-pass function, possibly infinite dimensional), $N_{o} \in \mathcal{H}^{\infty}\left(\mathbb{C}_{+}\right)$is outer (minimum phase, possibly infinite dimensional) and $m_{d}$ is finite dimensional and inner.

Based on the results in $[28,32]$ and [29], the optimal $\mathcal{H}^{\infty}$ controller is given by

$C_{o p t}(s)=E_{\gamma_{o}}(s) m_{d}(s) \frac{N_{o}^{-1}(s) F_{\gamma_{o}}(s) L(s)}{1+m_{n}(s) F_{\gamma_{o}}(s) L(s)}$,

where $E_{\gamma}(s)=\frac{W_{1}(-s) W_{1}(s)}{\gamma^{2}}-1$. Let the right half plane zeros of $E_{\gamma}(s)$ be $\beta_{i}, i=1, \ldots, n_{1}$, the right half plane poles of $P_{0}(s)$ be $\alpha_{k}, k=1, \ldots, l$ and that of $W_{1}(s)$ be $\eta_{i}, i=1, \ldots, n_{1}$. Then

$F_{\gamma}(s)=G_{\gamma}(s) \prod_{i=1}^{n_{1}} \frac{s-\eta_{i}}{s+\eta_{i}}$,

where $G_{\gamma}(s) \in \mathcal{H}^{\infty}\left(\mathbb{C}_{+}\right)$is minimum phase and determined from the following spectral factorization

$G_{\gamma}(s) G_{\gamma}(-s)=\left(1-\left(\frac{W_{2}(-s) W_{2}(s)}{\gamma^{2}}-1\right) E_{\gamma}(s)\right)^{-1}$.

Let $L(s)=\frac{L_{2}(s)}{L_{1}(s)}$, where $L_{1}(s)$ and $L_{2}(s)$ are polynomials with degrees $\leq\left(n_{1}+l-1\right)$, which are determined by the following interpolation conditions,

$0=L_{1}\left(\beta_{i}\right)+m_{n}\left(\beta_{i}\right) F_{\gamma}\left(\beta_{i}\right) L_{2}\left(\beta_{i}\right)$

$0=L_{1}\left(\alpha_{k}\right)+m_{n}\left(\alpha_{k}\right) F_{\gamma}\left(\alpha_{k}\right) L_{2}\left(\alpha_{k}\right)$

$0=L_{2}\left(-\beta_{i}\right)+m_{n}\left(\beta_{i}\right) F_{\gamma}\left(\beta_{i}\right) L_{1}\left(-\beta_{i}\right)$

$0=L_{2}\left(-\alpha_{k}\right)+m_{n}\left(\alpha_{k}\right) F_{\gamma}\left(\alpha_{k}\right) L_{1}\left(-\alpha_{k}\right)$,

for $i=1, \ldots, n_{1}$ and $k=1, \ldots, l$.

The optimal performance level $\gamma_{o}$ in (1), can be determined by finding the largest $\gamma$ value such that the spectral factorization (5) can be done and the interpolation conditions (6) are satisfied for some non-zero $L_{1}, L_{2}$. Once $\gamma_{o}$ is determined, the optimal $\mathcal{H}^{\infty}$ controller can be obtained by (3). An Matlab based computational package, as well as a guideline for the design of such systems, can be found in [33].

\section{Modeling of HDD servo systems}

A modern HDD can be considered as an ultra-high precision mechatronics device and its main components are illustrated in Fig. 1. For a single stage HDD servo system, the magnetic head is actuated by a VCM (Voice Coil Motor) and its position is measured by the PES (position error signal) demodulated from the embedded servo information on the magnetic disk. The dynamics of a single stage HDD servo system can be modeled as:

$P(s)=\frac{K_{D C} e^{-h s}}{s^{2}} T_{s}(s) T_{m}(s)$,

where $K_{D C}$ is the actuator DC gain, $h$ the total time delay from various sources such as PWM filters, power amplifiers, actuator delays, and $T_{s}(s)$ the first translational mode (system mode):

$T_{s}(s)=\frac{A_{s}(s)}{B_{s}(s)}=\frac{s^{2}+2 \xi_{z, 0} \omega_{n_{z, 0}} s+\omega_{n_{z, 0}}^{2}}{s^{2}+2 \xi_{p, 0} \omega_{n_{p, 0}} s+\omega_{n_{p, 0}}^{2}}$,

where $\xi_{z, 0}$ and $\omega_{n_{z, 0}}$ are the damping ratio and natural frequency of the zeros of the system mode, and $\xi_{p, 0}$ and $\omega_{n_{p, 0}}$ the damping ratio and natural frequency of the poles. Note that $T_{s}(s)$ is stable, 


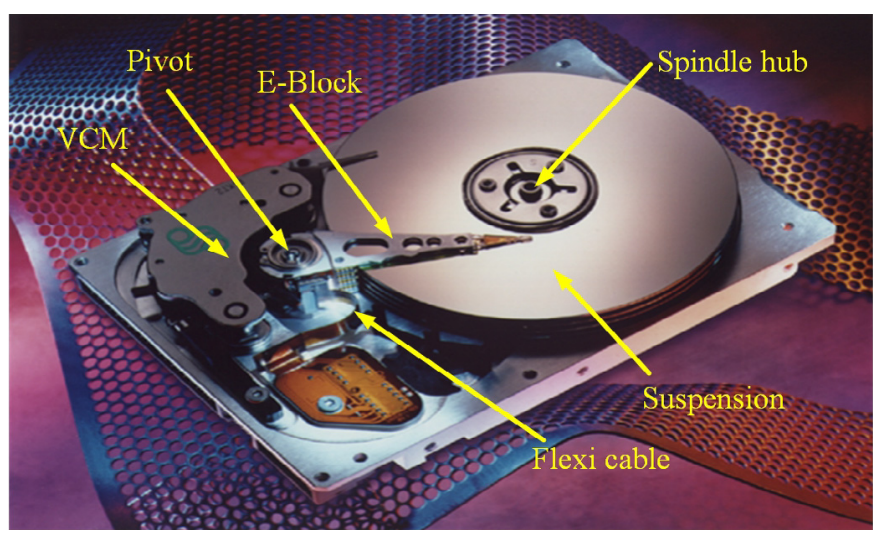

Fig. 1. Schematic of a modern HDD (by courtesy of Seagate).

biproper and minimum phase, i.e., $T_{s}(s) \in \mathcal{H}^{\infty}$ and $T_{s}^{-1}(s) \in \mathcal{H}^{\infty}$, which is usually guaranteed by the mechanical design of HSA (Head Suspension Assembly). All the high frequency resonant modes can be modeled by $T_{m}(s)$ :

$T_{m}(s)=\prod_{i=1}^{N} \frac{\frac{1}{\omega_{n_{z, i}}^{2}} s^{2}+2 \frac{\xi_{z, i}}{\omega_{n_{z, i}}} s+1}{\frac{1}{\omega_{n_{p, i}}^{2}} s^{2}+2 \frac{\xi_{p, i}}{\omega_{n_{p, i}}} s+1}$,

where $\xi_{z, i}, \xi_{p, i}, \omega_{n_{z, i}}$ and $\omega_{n_{p, i}}$ are the damping ratios and natural frequencies of the $i$ th resonant mode.

We define the nominal model of (7) as:

$P_{0}(s)=\frac{K_{0} e^{-h s}}{s^{2}} \bar{T}_{s}(s)$

where

$\bar{T}_{s}(s)=\frac{\bar{A}_{s}(s)}{\bar{B}_{s}(s)}=\frac{s^{2}+2 \bar{\xi}_{z, 0} \bar{\omega}_{n_{z, 0}} s+\bar{\omega}_{n_{z, 0}}^{2}}{s^{2}+2 \bar{\xi}_{p, 0} \bar{\omega}_{n_{p, 0}} s+\bar{\omega}_{n_{p, 0}}^{2}}$,

represents the nominal system mode, and $K_{0}$ is the nominal DC gain. Note that the DC gain $K_{D C}$ of (7) is usually calibrated in factory tests of HDD manufacturing. Therefore we will assume $K_{0}=K_{D C}$ in the rest of the paper.

As depicted in Fig. 2, the nominal plant $P_{0}(s)$ is illustrated by the thick blue line and the actual plant with various uncertainties (and high frequency resonance modes) can be illustrated by the magenta lines.

Now that the multiplicative uncertainties can be written as:

$\Delta P_{m}(s)=\frac{P(s)-P_{0}(s)}{P_{0}(s)}=\frac{A_{s}(s) \bar{B}_{s}(s)}{\bar{A}_{s}(s) B_{s}(s)} T_{m}(s)-1$.

If we denote the multiplicative uncertainty bound $W_{2}(s)$ with $\left|\Delta P_{m}(s)\right|_{s=j \omega} \leq\left|W_{2}(s)\right|_{s=j \omega}$, the upper and lower bounds of all possible plant variations can be estimated by $\left|P_{0}(j \omega)\right| \pm \mid P_{0}(j \omega)$ $W_{2}(j \omega) \mid$. This method will be used to determine the uncertainty bound $W_{2}(s)$ with plant measurement data, which will be detailed in the design case study.

\section{Mixed sensitivity optimization for infinite dimensional sys- tems}

Various robust control algorithms has been widely discussed for HDD servo design (see [4-6,17-19], to cite just a few contributions). Note that in track following mode, the HDD servo system needs to reject various disturbances such that the head can operate close enough to the track center. Therefore, the main control objective is to optimize the servo control loop
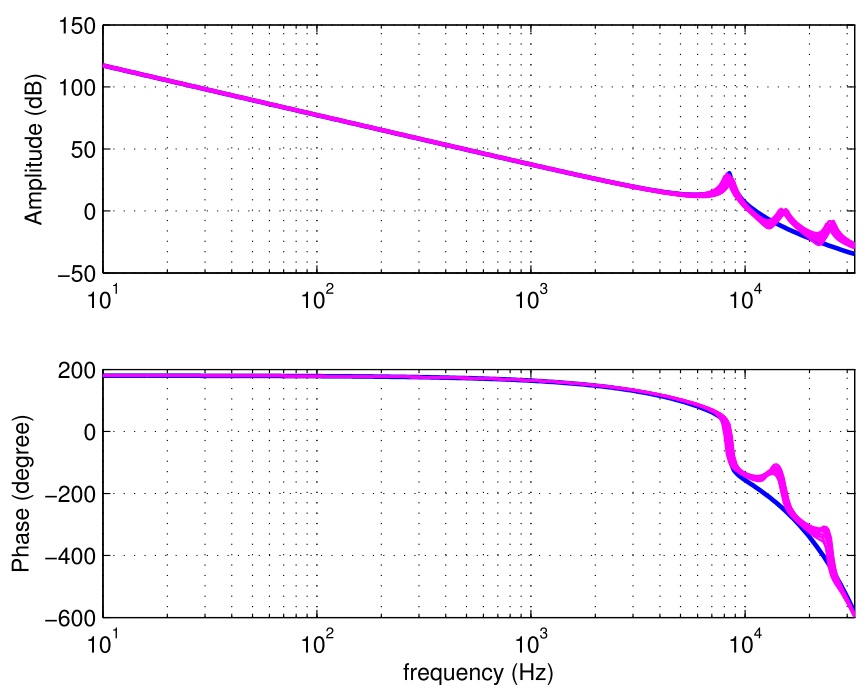

Fig. 2. Nominal plant and actual plant with uncertainties . (For interpretation of the references to color in this figure legend, the reader is referred to the web version of this article.)

to compromise the rejection capability and model uncertainties. An applicable $\mathcal{H}^{\infty}$ control method in servo loop shaping is mixed sensitivity optimization [19,34], where the nominal performance (determined by performance weighting $W_{1}(s)$ ) and robust stability (determined by uncertainty weighting $W_{2}(s)$ ) can be optimized simultaneously:

$\inf _{\text {Cstab. } P_{0}}\left\|\left[\begin{array}{c}W_{1}(s)\left(1+P_{0}(s) C(s)\right)^{-1} \\ W_{2}(s) P_{0}(s) C(s)\left(1+P_{0}(s) C(s)\right)^{-1}\end{array}\right]\right\|_{\infty}$.

Note that standard $\mathcal{H}^{\infty}$ control theory, as readily implemented in the robust control toolbox of Matlab, cannot be applied to this particular problem, due to the existence of time delays in $P_{0}(s)$. In what follows, we would like to investigate the mixed sensitivity optimization problem (13) using the robust control theory for infinite dimensional systems [28] and [32].

To begin with, we denote

$\begin{aligned} \tilde{P}_{0}(s) & =P_{0}(s) * \bar{T}_{s}^{-1}(s) \approx \frac{K_{0} e^{-h s}}{(s+\epsilon)^{2}}, \\ \tilde{C}(s) & =C(s) * \bar{T}_{s}(s),\end{aligned}$

where $\epsilon>0$ is sufficiently small. Recall the fact that $\bar{T}_{s}(s) \in \mathcal{H}^{\infty}$ and $\bar{T}_{s}^{-1}(s) \in \mathcal{H}^{\infty}$. Now the original $\mathcal{H}^{\infty}$ optimization (13) can be written as:

$\inf _{\tilde{C} s t a b . \tilde{P}_{0}}\left\|\left[\begin{array}{c}W_{1}(s)\left(1+\tilde{P}_{0}(s) \tilde{C}(s)\right)^{-1} \\ W_{2}(s) \tilde{P}_{0}(s) \tilde{C}(s)\left(1+\tilde{P}_{0}(s) \tilde{C}(s)\right)^{-1}\end{array}\right]\right\|_{\infty}$,

where the optimal $\mathcal{H}^{\infty}$ controller, denoted by $\tilde{C}_{\text {opt }}(s)$, yields:

$\left\|\left[\begin{array}{c}W_{1}(s)\left(1+\tilde{P}_{0}(s) \tilde{C}_{o p t}(s)\right)^{-1} \\ W_{2}(s) \tilde{P}_{0}(s) \tilde{C}_{o p t}(s)\left(1+\tilde{P}_{0}(s) \tilde{C}_{o p t}(s)\right)^{-1}\end{array}\right]\right\|_{\infty}=\gamma_{o}$,

with $\gamma_{0}$ the optimal $\mathcal{H}^{\infty}$ performance index.

For disturbance attenuation purposes, the sensitivity of HDD servo tracking loop is usually designed with low frequency rolloff of $40 \mathrm{~dB} / \mathrm{dec}$ (if not more). Therefore we select $W_{1}(s)=K_{1} / s^{2}$ to shape the low frequency feature. Meanwhile the multiplicative uncertainty weighting is chosen as $W_{2}(s)=a+b s+c s^{2}$ to accommodate model uncertainties.

Observe that $\tilde{P}_{0}(s)$ admits the coprime inner/outer factorization $\tilde{P}_{0}(s)=m_{n}(s) N_{o}(s)$, where $m_{n}(s)=e^{-h s} \in \mathcal{H}^{\infty}\left(\mathbb{C}_{+}\right)$is inner and $N_{o}(s)=K_{0} /(s+\epsilon)^{2} \in \mathcal{H}^{\infty}\left(\mathbb{C}_{+}\right)$is outer. Recall the 
$\mathcal{H}^{\infty}$ controller design procedure for infinite dimensional systems described in Section 2. The optimal solution of (15) can be derived as

$\tilde{C}_{o p t}(s)=\frac{1}{K_{0}} E_{\gamma_{0}}(s) \frac{s^{2} F_{\gamma_{0}}(s) L(s)}{1+e^{-h s} F_{\gamma_{0}}(s) L(s)}$,

where

$L(s)=\frac{n L(s)}{d L(s)}=\frac{1+a_{L} s}{1-a_{L} s}$,

with $a_{L}$ and $\gamma_{0}$ determined from interpolation conditions in (6). The functions $E_{\gamma_{0}}$ and $F_{\gamma_{0}}$ are defined as

$E_{\gamma_{0}}(s)=\frac{n E(s)}{d E(s)}=\frac{K_{1}^{2}-\gamma_{o}^{2} s^{4}}{\gamma_{o}^{2} s^{4}}$,

and $F_{\gamma_{0}}(s)$ is a stable transfer function in the form

$F_{\gamma_{0}}(s)=\frac{n F(s)}{d F(s)}=\frac{s^{2}}{f_{4} s^{4}+f_{3} s^{3}+f_{2} s^{2}+f_{1} s+f_{0}}$,

whose coefficients $f_{i}, i=0, \ldots 4$ depend on $\gamma_{0}$ and the coefficients of $W_{1}$ and $W_{2}$.

Thus, the controller parameters $\gamma_{0}, a_{L}$, and $f_{i}, i=0, \ldots 4$ can be determined numerically from the problem data $K_{1}, a, b, c$ and $h$, by deploying the Matlab-based program of [33] for the numerical design problem, as detailed in Section 2.

\subsection{Controller structure}

It is noticed that the $\mathcal{H}^{\infty}$ controller (16) has unstable zero-pole cancelations due to the interpolation conditions [32]. However the exact cancelations for the factorization terms are not possible because of the time delay in the controller. We would like to rearrange the terms in the controller to eliminate the unstable zero-pole cancelations such that the above controller structure is implementable in a stable manner. A similar controller structure has also been obtained in [35].

Based on the observations made in [29], $\tilde{C}_{o p t}(s)$ can be rewritten as:

$\tilde{C}_{\text {opt }}(s)=\frac{1}{K_{0} f_{4}}\left(\frac{G(s)}{1+G(s) H_{F I R}(s)}\right)$,

where

$G(s)=\frac{1+a_{L} s}{1+Q_{G}+a_{L} s}, \quad Q_{G}=\frac{2}{f_{4}} \frac{d F\left(-1 / a_{L}\right)}{n E\left(-1 / a_{L}\right)}$,

and

$H_{F I R}(s)=\mathcal{L}\{h(t)\}$,

with

$h(t)=\left\{\begin{array}{cl}C_{0} e^{A_{0} t} B_{0} & 0 \leq t \leq h \\ 0 & t>h\end{array}\right.$

where

$C_{0}\left(s I-A_{0}\right)^{-1} B_{0}=\frac{h_{0}(s)}{\left(s+1 / a_{L}\right) n E(s)}$,

and

$$
\begin{aligned}
h_{0}(s) & =\frac{1}{f_{4}}\left(1 / a_{L}-s\right) d F(s) \\
& -\left(1 / a_{L}+s\right) n E(s)-Q_{G} n E(s) .
\end{aligned}
$$

Note that $H_{F I R}(s)$ is a filter, whose impulse response is of finite duration (FIR filter). From (14), (18) (19) and (21), we can derive the optimal $\mathcal{H}^{\infty}$ controller

$C_{\text {opt }}(s)=\frac{1}{K_{0} f_{4}} \bar{T}_{s}^{-1}(s)\left(\frac{G(s)}{1+G(s) H_{F I R}(s)}\right)$,

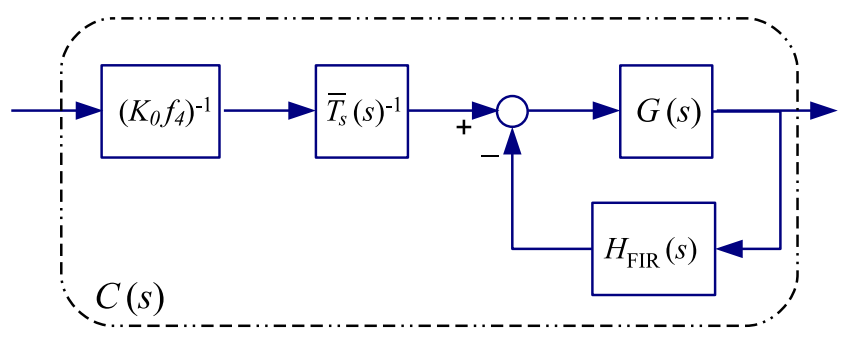

Fig. 3. Controller structure.

which can be implemented as depicted in Fig. 3. It is worth noting that the controller structure (22) facilitates stable digital implementations due to the FIR feature of $H_{F I R}(s)$ and the first order representation of $G(s)$.

\subsection{Digital implementation with $\mathcal{H}^{\infty}$ approximation}

In the digital implementation of the above $\mathcal{H}^{\infty}$ controller $C_{\text {opt }}(s)$ with the structure in (22), we cannot directly digitize the controller by discretization of each block in Fig. 3 at a specific sampling time $T_{s}$, mainly due to the existence of time delays in the FIR filter $H_{F I R}(s)$, which can cause controller stability issue when performing direct truncation of the sampled-data digital implementation. The problem will be more eminent when the sampling time $T_{S}$ is getting larger. In what follows, we would like to investigate the $\mathcal{H}^{\infty}$-based optimal discretization and approximation method for $H_{F I R}(s)$, by recalling the sampled-data FIR filter $\mathcal{H}^{\infty}$ optimization method discussed in [30] and [31].

Before conducting the $\mathcal{H}^{\infty}$ optimal approximation, we should determine a stable digital filter as the target of approximation without considering the constraint of FIR. To start with, we perform the following factorization on $H_{F I R}(s)$ :

$H_{F I R}(s)=H_{1}(s)+e^{-h s} \cdot H_{2}(s)$,

Then we can get its digital representation in IIR form by direct discretization with the sampling time of $T_{S}$,

$H_{I I R}(z)=H_{1}(z)+z^{-m} \cdot H_{2}(z), m=h / T_{s}$.

Then we apply zero-pole cancelation on the digital IIR filter $H_{I I R}(z)$ and obtain a stable and strictly proper transfer function $\hat{H}_{I I R}(z)$ (guaranteed by (20) and (21)) with the following zero-pole representation

$\hat{H}_{I I R}(z)=K_{h} \frac{\left(z-z_{1}\right) \cdots\left(z-z_{l}\right)}{\left(z-p_{1}\right) \cdots\left(z-p_{q}\right)}, l \leq q ; l, q \in \mathbb{N}^{+}$,

Now we would like to employ discrete time $\mathcal{H}^{\infty}$ optimization method design an optimal digital FIR filter $H_{o p t}(z)$ to approximate the digital IIR filter $\hat{H}_{I I R}(z)$. We assume that the minimal realization of the digital FIR filter $H_{o p t}(z)$ with length of $M$ can be expressed as:

$H_{\text {opt }}(z)=\sum_{k=0}^{M-1} a_{k} z^{-k}:=\left[\begin{array}{c:c}A_{H} & B_{H} \\ \hdashline C_{H} & D_{H}\end{array}\right]$,

where

$A_{H}=\left[\begin{array}{ccccc}0 & 1 & 0 & \cdots & 0 \\ \vdots & \ddots & \ddots & & \vdots \\ \vdots & & \ddots & \ddots & 0 \\ \vdots & & & \ddots & 1 \\ 0 & \cdots & \cdots & \cdots & 0\end{array}\right], B_{H}=\left[\begin{array}{c}0 \\ \vdots \\ \vdots \\ 0 \\ 1\end{array}\right]$, 
Table 1

Model coefficients.

\begin{tabular}{llllllllll}
\hline$K_{0}$ & $h$ & $\bar{\xi}_{z, 0}$ & $\bar{\xi}_{p, 0}$ & $\bar{\omega}_{n_{z, 0}}$ & $\bar{\omega}_{n_{p, 0}}$ & $\epsilon$ & $K_{1}$ & $a$ & $b$ \\
\hline $5.2269 \times 10^{8}$ & $6 \times 10^{-5}$ & 0.99 & 0.018 & $1.244 \times 10^{5}$ & $5.29 \times 10^{4}$ & 0.01 & $2 \times 10^{7}$ & 0.3125 & $9.4211 \times 10^{-6}$ \\
\hline
\end{tabular}

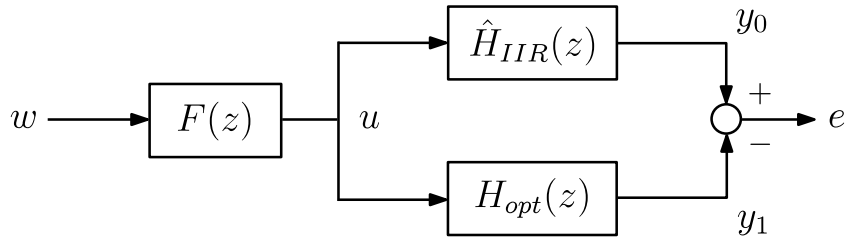

Fig. 4. Error system block diagram.

$C_{H}=\left[\begin{array}{llll}a_{M-1} & a_{M-2} & \cdots & a_{1}\end{array}\right], D_{H}=a_{0}$.

We can now propose an $\mathcal{H}^{\infty}$-based approximation problem as depicted in Fig. 4, where the error system from $w$ to $e$ can be written as:

$E(z)=\left[\hat{H}_{I I R}(z)-H_{o p t}(z)\right] \cdot F(z)=\left[\begin{array}{c:c}A_{E} & B_{E} \\ \hdashline C_{E}(\overrightarrow{\mathbf{a}}) & D_{E}(\overrightarrow{\mathbf{a}})\end{array}\right]$,

$\overrightarrow{\mathbf{a}}=\left[\begin{array}{llll}a_{M-1} & a_{M-2} & \cdots & a_{0}\end{array}\right]^{T} \in \mathbb{R}^{M}$,

where a weighting transfer function $F(z)$ is introduced to penalize the error between $\hat{H}_{I I R}(z)$ and $H_{\text {opt }}(z)$. In particular, $F(z)$ is chosen to be a stable, real-rational, and strictly proper low-pass transfer function with adjustable cutoff frequency to facilitate the $\mathcal{H}^{\infty}$ approximation.

The optimization problem is to find the optimal FIR filter with length of $M \in \mathbb{N}^{+}$and filter coefficient vector of $\overrightarrow{\mathbf{a}} \in \mathbb{R}^{M}$ to minimize the $\mathcal{H}^{\infty}$ norm of the sampled-data error system $E(z)$. Based on the results of [30] and [31], the $\mathcal{H}^{\infty}$ optimization problem can be formulated as a convex optimization problem using the KYP (Kalman-Yakubovich-Popov) lemma, and solved by LMIs (linear matrix equality). In particular, we can use the following steps to the optimal length $M$ and the optimal filter coefficient vector $\overrightarrow{\mathbf{a}}$ :

Step 1 Determine a reasonable searching range of $\left[M_{\min }, M_{\max }\right]$ with $M_{\min }$ and $M_{\max }$ the minimal and maximal integer numbers to search, and let $M=M_{\min }$ initially.

Step 2 Given the filter length $M$, find $\overrightarrow{\mathbf{a}} \in \mathbb{R}^{M}$ to minimize $\gamma_{M}=\|E(z)\|_{\infty}$ subject to the following LMIs

$$
\left[\begin{array}{ccr}
A_{E}^{T} X A_{E}-X & A_{E}^{T} X B_{E} & C_{E}^{T}(\overrightarrow{\mathbf{a}}) \\
* & B_{E}^{T} X B_{E}-\gamma_{M} I & D_{E}^{T}(\overrightarrow{\mathbf{a}}) \\
* & * & -\gamma_{M} I
\end{array}\right]<0,
$$

Step 3 Repeat the above step with all positive integers $M \in$ $\left[M_{\min }, M_{\max }\right]$ and find the minimal $\gamma_{o p t} \in\left\{\gamma_{1}, \ldots, \gamma_{M_{\max }}\right\}$, which determines the optimal FIR filter length $M_{\text {opt }}$ and coefficients $\overrightarrow{\mathbf{a}}_{\text {opt }}$. Then the optimal sampled-data FIR filter $H_{o p t}(z)$ can be obtained from (26).

The above optimization problem can be efficiently solved by numerical softwares such as SeDuMi or YALMIP. Then the digital implementation of the $\mathcal{H}^{\infty}$ controller $C_{\text {opt }}(s)$ with the structure in (22) can be derived as

$C_{\text {opt }}(z)=\frac{1}{K_{0} f_{4}} \bar{T}_{s}^{-1}(z)\left(\frac{G(z)}{1+G(z) H_{o p t}(z)}\right)$, where $\bar{T}_{s}^{-1}(z)$ and $G(z)$ can be computed by discretizing $\bar{T}_{s}^{-1}(s)$ and $G(s)$ with the sampling time $T_{s}$. More details on digital implementation will be given in the next section with a case study.

Remark 1. Note that the controller parameters are determined by the nominal plant and the weighting functions $W_{1}(s)$, and $W_{2}(s)$. In industrial applications, the nominal plant can be obtained by DFT (Discrete Fourier Transform) based system identification method on representative drives. The performance weighting function is designed by the HDD low frequency disturbance rejection requirements, which is usually selected as $W_{1}(s)=$ $K_{1} / s^{2}$ to represent $40 \mathrm{~dB} / \mathrm{dec}$ roll-off at low frequency for the closed loop sensitivity. The uncertainty weighting function $W_{2}(s)$ is selected based on allowable variations of a disk drive product, where the parameters can be numerically determined such that the upper and lower bounds of all possible plant variations should be within the magnitude range between $\left|P_{0}(j \omega)\right| \pm\left|P_{0}(j \omega) W_{2}(j \omega)\right|$, which will be illustrated in Fig. 5 .

Remark 2. To Determine a reasonable searching range of $\left[M_{\min }\right.$, $\left.M_{\text {max }}\right]$ in Step 1 to find the digital FIR $H_{o p t}(z)$, we can first recall the original continuous time FIR $H_{F I R}(s)$ in (20) and (20), whose impulse response is of finite duration $h$. Therefore, it has at least $\left\lceil h / T_{s}\right\rceil$ steps in digital implementation with a sampling time of $T_{s}$. Therefore we can select $M_{\min }=\left\lceil h / T_{s}\right\rceil$, and choose $M_{\max }$ 3-6 times larger than $M_{\min }$ to start with.

\subsection{Padé approximation methods for comparison}

Most of existing results on HDD servo loop shaping employ low order Padé approximations (33) to simplify the problem to a finite dimensional system, with which the closed form solutions of $\mathcal{H}^{\infty}$ controllers to the problem (13) can be synthesized by standard 2-block $\mathcal{H}^{\infty}$ optimization [34].

$e^{-h s} \approx T_{h}^{m}(s):=\frac{1-k_{1} s+k_{2} s^{2}+\cdots \pm k_{m} s^{m}}{1+k_{1} s+k_{2} s^{2}+\cdots+k_{m} s^{m}}$,

where $T_{h}^{m}(s)$ is the $m$ th order Padé approximation.

Now that an important open problem is to explore the conservativeness of this method using various Padé approximations, and how they are compared with the direct method discussed above. For this purpose, we consider the mixed sensitivity optimization (13) by Padé approximations with $e^{-h s} \approx T_{h}^{m}(s), m=1,2,3$, respectively. We denote

$P_{0}^{m}(s)=\frac{K_{0}}{s^{2}} T_{h}^{m}(s) \bar{T}_{s}(s), \quad m=1,2,3$,

and

$\tilde{W}_{1}(s):=\frac{K_{1}}{\left(s+\epsilon_{1}\right)^{2}} \approx W_{1}(s)$,

$\tilde{W}_{2}(s):=\frac{a+b s+c s^{2}}{\left(\epsilon_{2} s+1\right)^{2}} \approx W_{2}(s)$.

A standard mixed sensitivity optimization problem can be formulated for finite dimensional models $P_{0}^{m}(s), m=1,2,3$,

$\inf _{C^{m}{ }^{m} \text { stab. } P_{0}^{m}}\left\|\left[\begin{array}{c}\tilde{W}_{1}\left(1+P_{0}^{m} C^{m}\right)^{-1} \\ \tilde{W}_{2} P_{0}^{m} C^{m}\left(1+P_{0}^{m} C^{m}\right)^{-1}\end{array}\right]\right\|_{\infty}$,

and it can be solved using Matlab's mixsyn command. 


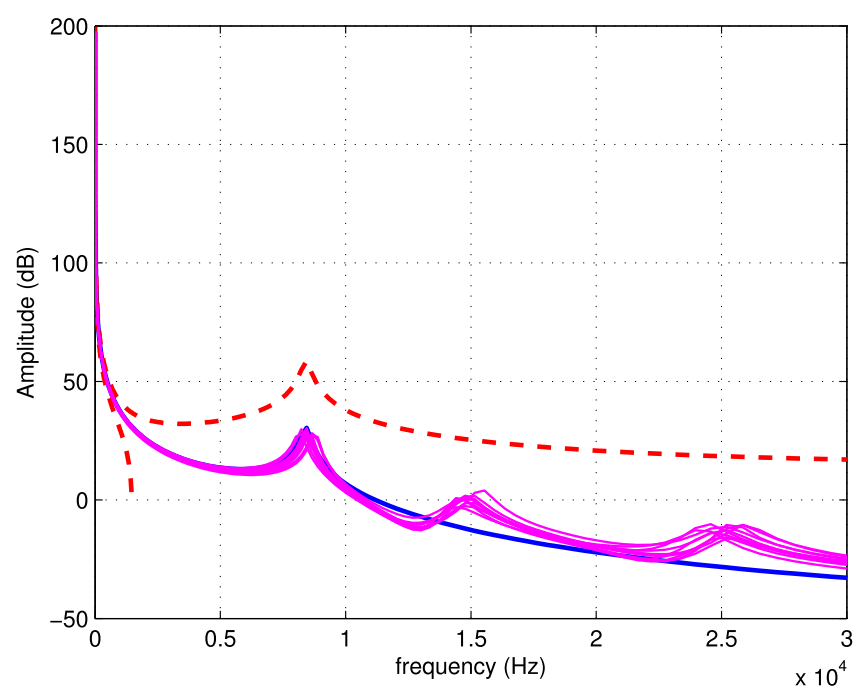

Fig. 5. Plant model and uncertainty bounds.

Note that low order Padé approximations result in inaccuracies at high frequency, while higher order approximations lead to high order controller dynamics and undesired zeros/poles which could cause transient problem. We will use a design example to compare the direct method for infinite dimensional systems and the Padé approximation methods.

\section{A design case study}

In this section, we apply the control algorithm derived in the above section to an industry design case, where an enterprise class 2.5-inch form factor HDD is considered. The HDD has $15 k R P M$ rotation speed and SAS (Serial Attached SCSI) interface with very high performance requirements for enterprise storage purposes, which has challenging design specifications on servo control. The tracking loop shape has to be highly optimized to address the mechanical resonances and windage disturbances at relatively high frequencies, induced by the highest rotation speed in the industry. The servo system of the HDD is operating with a sampling time of $T_{s}=1.5 \times 10^{-5}$. Note that the high performance enterprise class HDD has a dual-processor configuration on the servo electronics, where a DSP processor is fully dedicated to servo algorithms by executing fixed point representations of the controllers. With this, the overall servo architecture can support fast computations of complex control algorithms within the desired sampling time.

Based on HDD structure measurements, the nominal plant model (10) and the weighting functions can be determined in Table 1 . The nominal model (blue line) and the drive measurement data (magenta lines) of the HDD VCM structures are depicted in Fig. 2. The parameters of the uncertainty weighting function $W_{2}(s)$ are selected such that the corresponding uncertain bounds can cover the variations of all plant data, as depicted in Fig. 5.

Now we can perform the coprime inner/outer factorization described in Section 4, and follow the numerical tools of [33] to determine the optimal $\mathcal{H}^{\infty}$ performance index $\gamma_{\text {opt }}$ by numerically plotting the smallest singular value of the matrix representation of the interpolation equations [32]. As depicted in Fig. 6, $\gamma_{0}$ is the largest value for which the plot shows a zero, $\gamma_{0}=0.48336$.

The tools of [33] also give us $E_{\gamma_{0}}, F_{\gamma_{0}}$ and $L(s)$ defined in Section 4. More precisely, the following numerical values are computed

$E_{\gamma_{0}}(s)=\frac{n E}{d E}=\frac{-0.2336 s^{4}+4 \times 10^{14}}{0.2336 s^{4}}$,

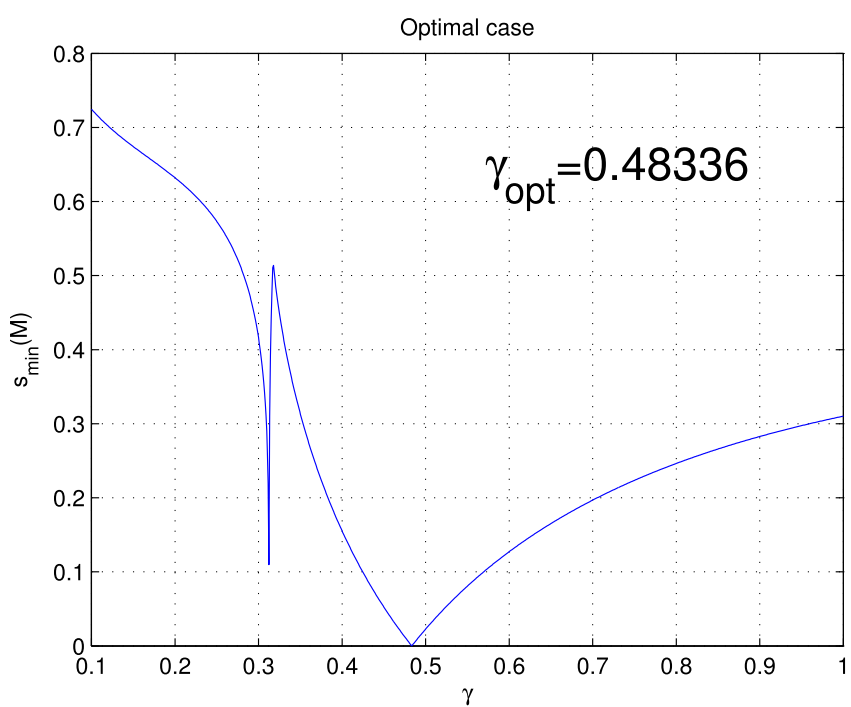

Fig. 6. Computation of $\gamma_{0}$.

$L(s)=\frac{n L}{d L}=\frac{1+a_{L} s}{1-a_{L} s}, \quad a_{L}=2.5124 \times 10^{-4}$,

and $f_{4}=1.81 \times 10^{-10}, f_{3}=2.127 \times 10^{-5}, f_{2}=0.8467, f_{1}=7294$, $f_{0}=3.156 \times 10^{7}$ in (17). Then the optimal $\mathcal{H}^{\infty}$ controller can be written as:

$C_{o p t}(s)=\Psi(s)\left(\frac{G(s)}{1+G(s) H_{F I R}(s)}\right)$,

where

$\Psi(s)=\frac{10.57 s^{2}+20100 s+2.96 \times 10^{10}}{s^{2}+246000 s+1.55 \times 10^{10}}$,

and

$G(s)=\frac{s+3.98 \times 10^{3}}{s+3.98 \times 10^{3}+4.4 \times 10^{5}}$,

and the FIR term $H_{F I R}(s)$ is determined by (20) and (21), with the impulse response (red line) depicted in Fig. 8.

Once obtaining the $\mathcal{H}^{\infty}$ optimal controller $C_{\text {opt }}(s)$ in continuous time domain, we would like to perform digital implementation based on the discussions in Section 4.2. The factorization on $H_{\text {FIR }}(s)$ in (23) can be computed as

$$
\begin{aligned}
& H_{F I R}(s)=H_{1}(s)+e^{-h s} \cdot H_{2}(s) \\
= & \frac{-33.068(s-16800)(s+3981)\left(s^{2}+120.8 s+305700\right)}{(s-6432)(s+6432)(s+3981)\left(s^{2}+4138000\right)} \\
& +\frac{-e^{-6 \times 10^{-5} s} \cdot 55.103 s^{2}(s+3981)}{(s-6432)(s+6432)(s+3981)\left(s^{2}+4138000\right)} .
\end{aligned}
$$

Then we can derive the digital IIR filter $\hat{H}_{I I R}(z)$ by direct discretization (24) with the sampling time of $T_{s}$, and zero-pole cancelation (25), where unstable poles of $p=1.1013$ and $p=$ $0.9953 \pm 0.0963 i$ are canceled

$$
\begin{aligned}
& H_{I I R}(z)=\frac{-4.34(z+0.459)(z-0.907)(z-0.910)(z-0.938)}{z^{4}(z-0.944)(z-0.940)} \\
& \frac{(z-0.938)(z-0.945)\left(z^{2}-1.99 z+0.999\right)\left(z^{2}+0.25 z+0.31\right)}{(z-0.909)(z-0.907)\left(z^{2}-1.99 z+0.999\right)}
\end{aligned}
$$

We select the digital weighting function

$F(z)=\frac{1}{z-3.059 \times 10^{-7}}$, 


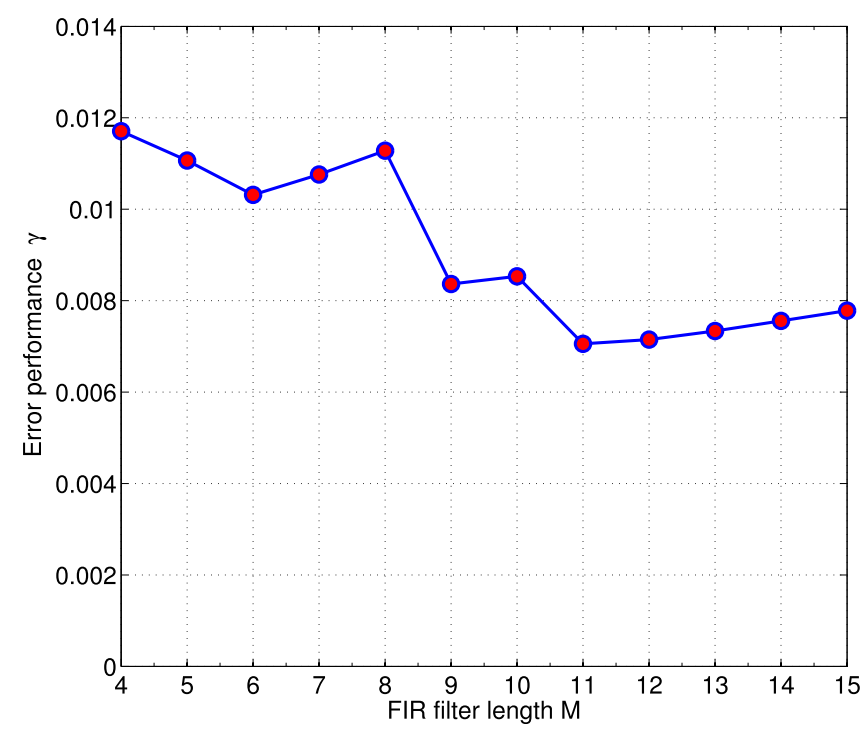

Fig. 7. $\mathcal{H}^{\infty}$ performance with FIR filter length $M$.

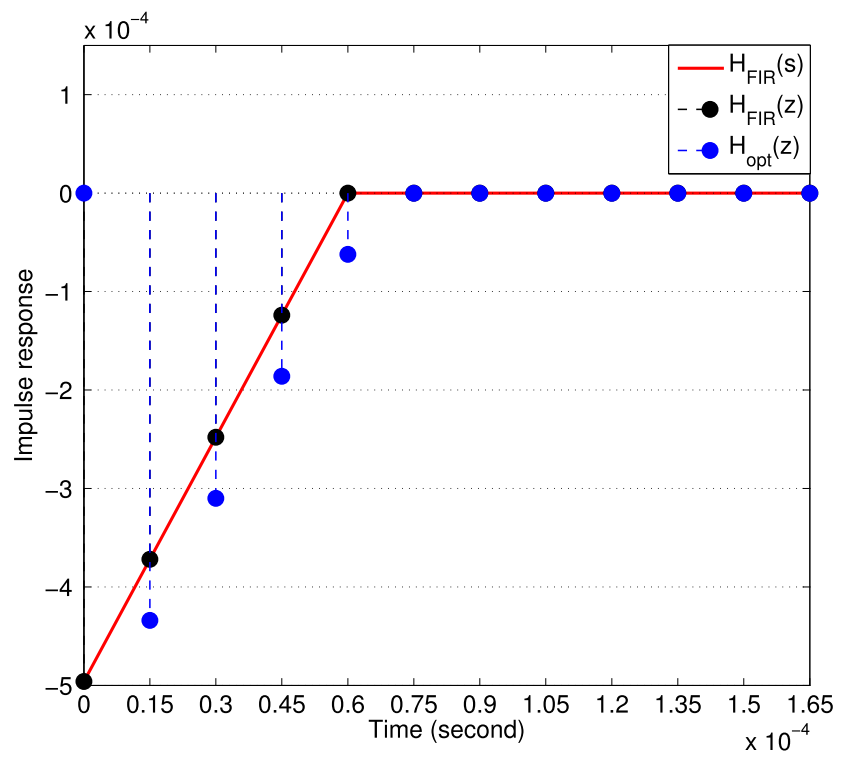

Fig. 8. Impulse responses of $H_{F I R}(s)$ and digital filters . (For interpretation of the references to color in this figure legend, the reader is referred to the web version of this article.)

and perform the $\mathcal{H}^{\infty}$ optimization procedure for digital FIR filter approximation as discussed in Section 4.2. The $\mathcal{H}^{\infty}$ performance indices $\gamma_{M}$ of the error system with different filter length $M$ can be depicted in Fig. 7, where the FIR filter with $M=11$ gives the minimum value of $\gamma_{\min } \approx 7.10 \times 10^{-3}$. Then we can fix the FIR filter length with $M=11$ and calculate the $\mathcal{H}^{\infty}$ optimal FIR filter $H_{\text {opt }}(z)$ based on the LMI optimization in (31),

$H_{\text {opt }}(z)=-4.546 e-5-4.34 z^{-1}-3.1 z^{-2}-1.861 z^{-3}$

$-0.6218 z^{-4}-0.002059 z^{-5}-0.002033 z^{-6}-0.001971 z^{-7}$

$-0.00188 z^{-8}-0.001776 z^{-9}-0.001732 z^{-10}$

$-0.002182 z^{-11}$

For comparison purposes, we also design the digital FIR filter $H_{F I R}(z)$ based on direct truncation of the impulse response of $H_{F I R}(s)$, without optimal approximation. As depicted in Fig. 8 the

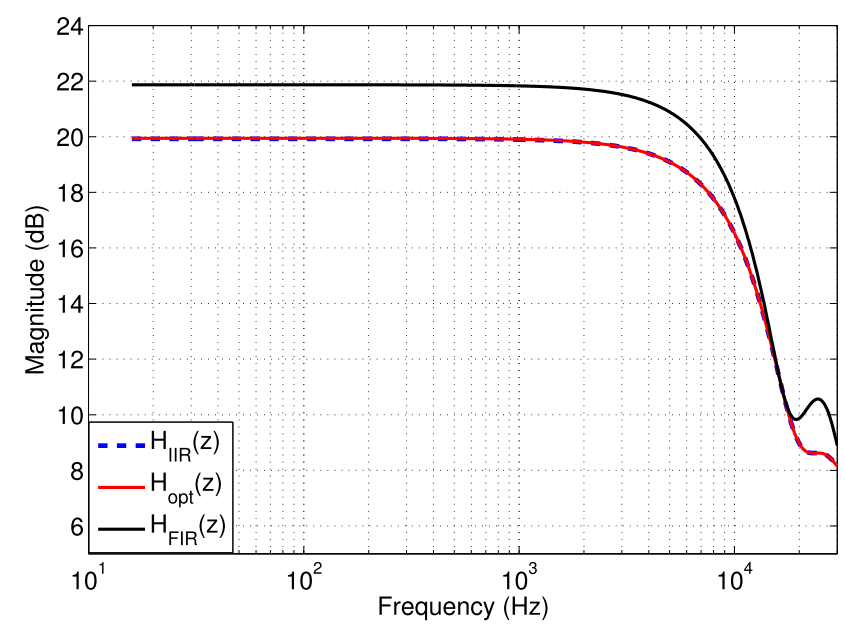

Fig. 9. Gains of digital filters.

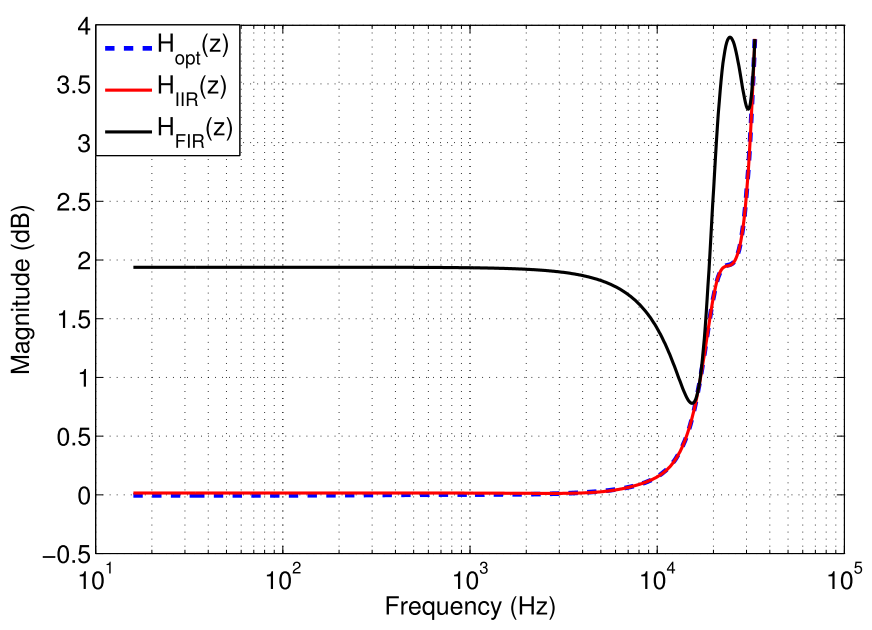

Fig. 10. Errors between $H_{F I R}(s)$ and the digital filters.

impulse response of the truncated FIR filter $H_{F I R}(z)$ (black dots) overlaps with that of $H_{\text {FIR }}(s)$ (red line), while the $\mathcal{H}^{\infty}$ optimal FIR filter $H_{\text {opt }}(z)$ (blue dots) adjusts its impulse response differently. Meanwhile the Magnitude responses of the above digital filters are plotted in Fig. 9, where it is clearly observed that $H_{F I R}(z)$ shows large differences from $H_{I I R}(z)$ (the "ideal" digital representation of $H_{F I R}(s)$ ), while $H_{\text {opt }}(z)$ and $H_{I I R}(z)$ are quite similar in terms of magnitude responses. It is also interesting to show the approximation performances of the above digital filters, compared with $H_{F I R}(s)$. Therefore the magnitude responses of the errors between the analog filter $H_{F I R}(s)$ and digital filters of $H_{I I R}(z), H_{F I R}(z)$ and $H_{o p t}(z)$ are computed, as depicted in Fig. 10. It is very clear that the $\mathcal{H}^{\infty}$ optimal FIR filter $H_{\text {opt }}(z)$ agrees well with $H_{F I R}(s)$, particularly at low frequencies. However, the direct truncation based filter $H_{F I R}(z)$ shows significant errors compared with the analog FIR filter $H_{F I R}(s)$, which explains the root cause of stability issue when $H_{F I R}(z)$ is used for controller digital implementations.

After deriving the optimal digital FIR filter $H_{o p t}(z)$, we can readily get the digital implementation of the $\mathcal{H}^{\infty}$ controller, $C_{\text {opt }}(z)$, by recalling (32). For comparison purpose, we also synthesize $\mathcal{H}^{\infty}$ controllers $C_{\text {padé }}^{m}(s)(m=1,2,3)$ based on (35) using various Padé approximations, and obtain their discrete time domain representations of $C_{\text {padé }}^{m}(z)(m=1,2,3)$ using foh (First Order Hold) based discretization method. 


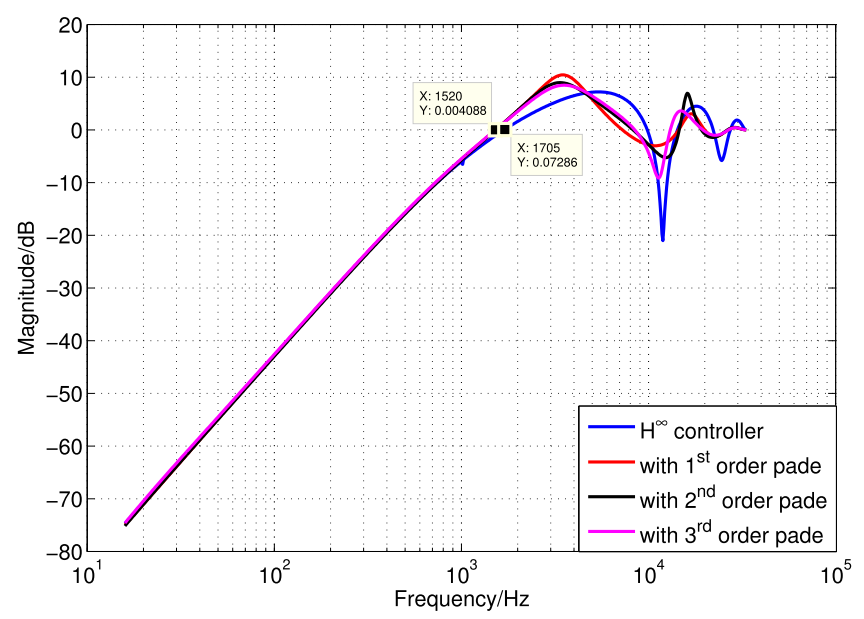

Fig. 11. Sensitivity comparisons.
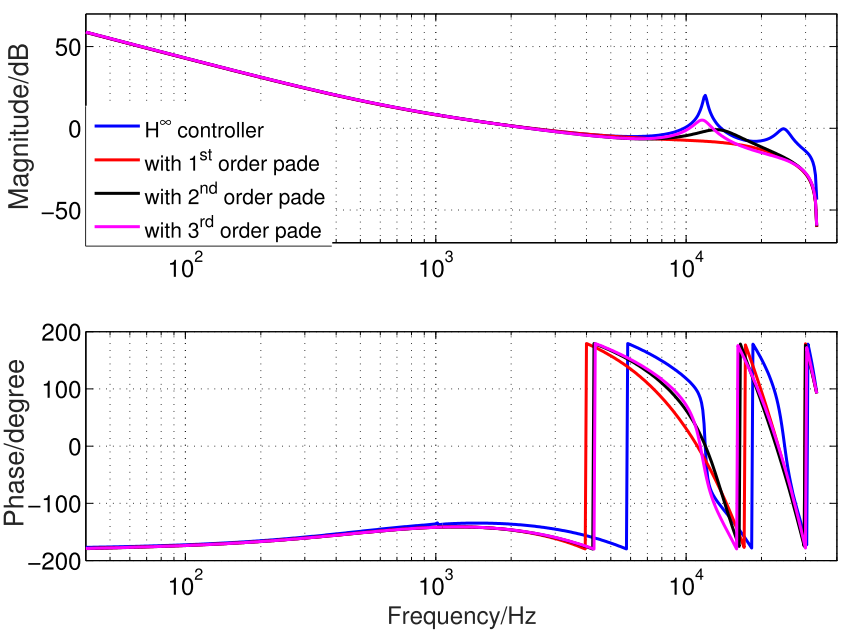

Fig. 12. Open loop bode comparisons.

Now that we can compare the time domain and frequency domain performances of the various controllers. As depicted in Fig. 11, the proposed method significantly improves closed loop bandwidth by more than $170 \mathrm{~Hz}$ compared to Padé approximation methods, with an additional advantage of a lower sensitivity peaking. It is also obvious that the proposed controller has more attenuation for disturbances below $5 \mathrm{kHz}$ due to lower sensitivity magnitude. Meanwhile the open loop bode plots are depicted in Fig. 12, where the gain/phase margins for the controllers can be seen clearly. The infinite dimensional controller designed in the present paper achieves a phase margin improvement of more than 10 degrees, which is consistent with the closed loop results in Fig. 11.

It is worth noting that the transient response of the infinite dimensional $\mathcal{H}^{\infty}$ controller also outperforms the Padé approximation methods. By taking a step response, we can observe that the proposed method in present paper demonstrates much smaller overshoot and less oscillations during settling (as depicted in Fig. 13).

\section{HDD TMR analysis}

In this section, we investigate the tracking TMR and tracking TPI capability of the designed servo loop, by applying industry measured PES data from field. In HDD industries, the major RRO

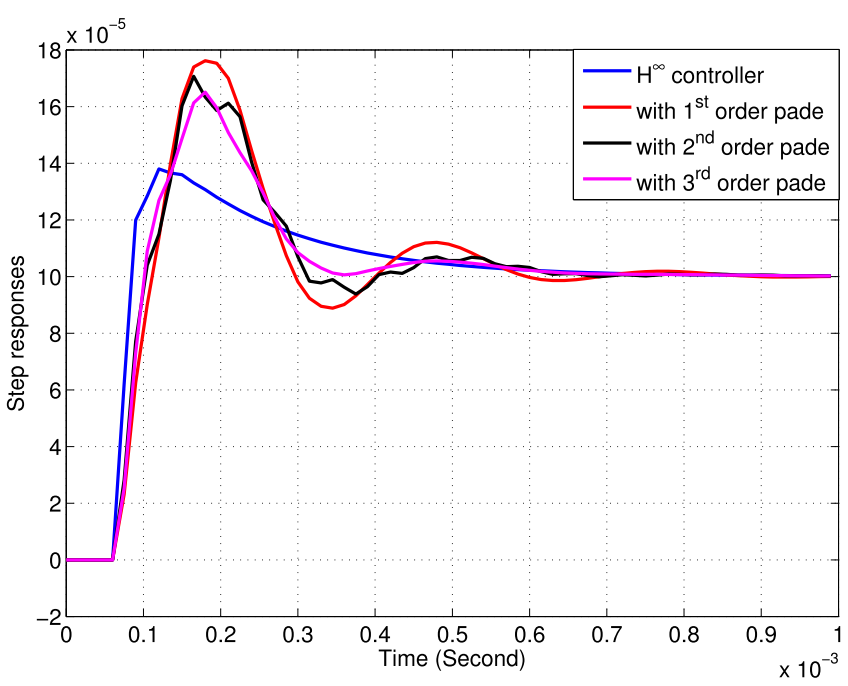

Fig. 13. Comparisons of step responses. (For interpretation of the references to color in this figure legend, the reader is referred to the web version of this article.)

(Repeatable Runout) components of PES (Position Error Signal) are usually treated separately. Therefore the TMR analysis is based on the NRRO components of PES data. The performance of algorithms is evaluated on HDD track following mode, where multiple tracks from Outer Diameter (OD), Middle Diameter (MD) and Inner Diameter (ID))locations are selected to reflect the tracking capability across the disk surface. The external conditions for the performance evaluation are based on the common standards in HDD industries, which can well represent the operating conditions for HDD.

Fig. 14 shows the NRRO spectrum with the $\mathcal{H}^{\infty}$ controller proposed in present paper and similar NRRO spectrum is also provided for the case of 1st order Padé approximation method, as illustrated in Fig. 15. It is very clear that the NRRO disk modes from $2 \mathrm{kHz}$ to $5 \mathrm{kHz}$ are better attenuated with the proposed controller, which agrees well with the loop shape comparisons in Fig. 11. We also decompose the drive NRRO data to frequency bins (with every $1000 \mathrm{~Hz}$ bandwidth up to $10 \mathrm{kHz}$ ) such that NRRO frequency components can be compared at given frequency ranges. As depicted in Fig. 16, the proposed infinite dimensional $\mathcal{H}^{\infty}$ control method outperforms the Padé based $\mathcal{H}^{\infty}$ method at all frequencies up to $6000 \mathrm{~Hz}$, which greatly improves tracking TMR due to the fact that major windage disturbances, vibrations and mechanical modes are more eminent at low frequency ranges.

A more detailed comparison is given in Table 2, where TMR statistics of Mean NRRO value and Mean $+3 \sigma$ NRRO are provided, with the corresponding tracking TPI capabilities (determined by industry specific statistical software developed by Seagate). It is shown that 2 nd order and 3rd order Padé approximation methods outperform 1st order one by more than $8 \%$ TPI improvement. But higher order Padé approximations will not help further. Note that major issues with higher order Padé approximations are (1) higher order controllers resulted from the models, and (2) undesired zeros/poles introduced by Padé approximations. As a matter of fact, a common practice in industry HDD servo control is still 1st order Padé approximation based method. The data in Table 2 clearly demonstrates the improvement using the $\mathcal{H}^{\infty}$ control method for infinite dimensional systems, developed in present paper, where more than 15\% TPI improvement can be achieved (see Fig. 15).

Recall the widely deployed PTOS track seeking control strategy, where the seek profiles are generated by approximating 


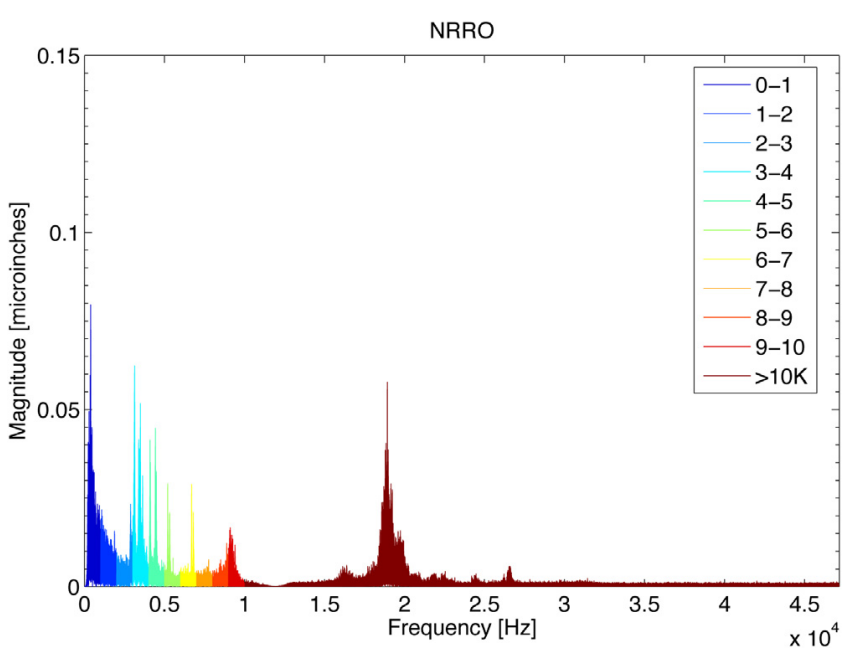

Fig. 14. Frequency components of NRRO: the proposed method.

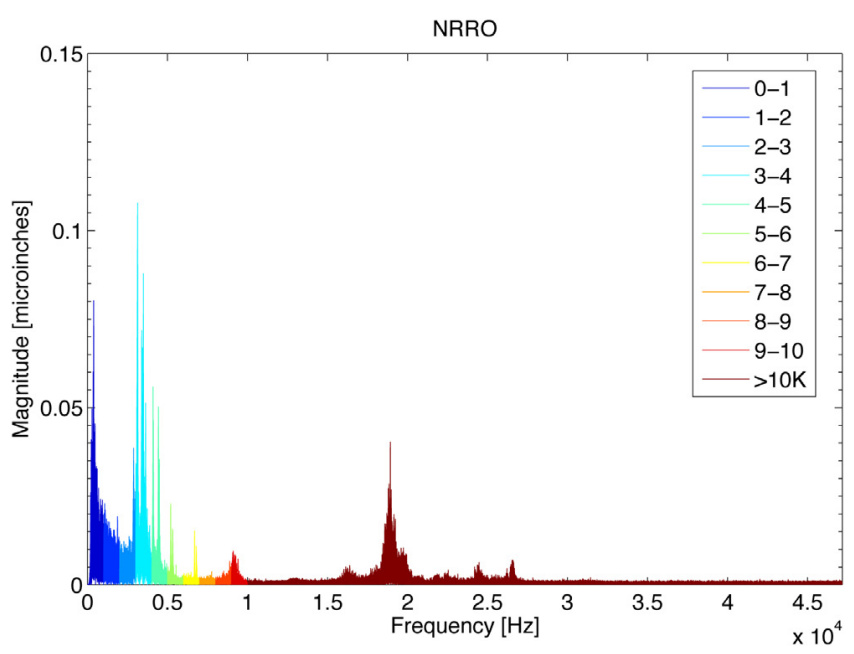

Fig. 15. Frequency components of NRRO: 1st order Padé approximation.

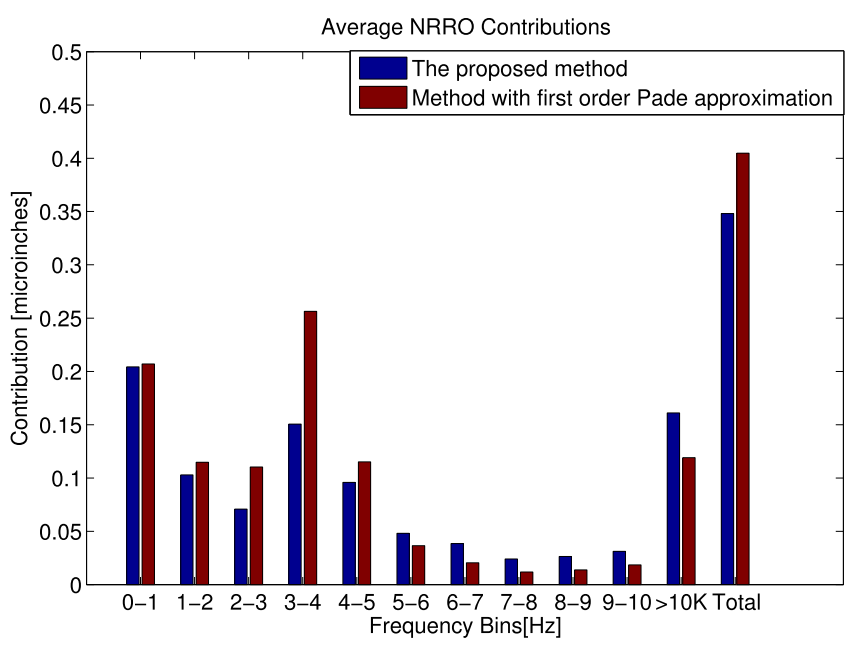

Fig. 16. Comparisons of NRRO frequency components.

Bang-Bang control for time optimization purpose (see for example, $[11,12,36]$ and $[6])$. Therefore, the significant improvements
Table 2

Performance comparisons.

\begin{tabular}{lllll}
\hline Methods & $\begin{array}{l}\text { NRRO: } \\
\text { Mean } \\
(\mu \text { in. })\end{array}$ & $\begin{array}{l}\text { NRRO: MeanTPI } \\
+3 \sigma \text { ( } \mu \text { in. })\end{array}$ & $\begin{array}{l}\text { TPI } \\
\text { improvement }\end{array}$ \\
\hline $\begin{array}{l}\text { 1st order Padé } \\
\text { approximation method }\end{array}$ & 0.40 & 0.59 & $193 K$ & N/A \\
$\begin{array}{l}\text { 2nd order Padé } \\
\text { approximation method }\end{array}$ & 0.37 & 0.55 & $209 K$ & $8.29 \%$ \\
$\begin{array}{l}\text { 3rd order Padé } \\
\text { approximation method } \\
\begin{array}{l}\text { Method of the present } \\
\text { paper }\end{array}\end{array}$ & 0.37 & 0.54 & $212 K$ & $8.96 \%$ \\
\hline
\end{tabular}

of transient responses using the proposed method (Fig. 13) indicates better track seeking performance. Particularly, the transient features of lower overshoot and less oscillations (refer to the blue line of Fig. 13) will result in better seek settling and thus higher data IOPS (IO-Per-Second). The quantified improvement of IOPS performance requires seek profiles and seek settling TMR, which deserves a separate study.

\section{Concluding remarks}

In this paper, we have applied a robust control technique for HDD head positioning, where the time delay of the model was treated as an infinite dimensional block directly, as opposite to the Padé approximation methods of existing results. The explicit form of the $\mathcal{H}^{\infty}$ controller was provided and an internal feedback structure of the controller was also given, which consists of a first order filter with a feedback consisting of an FIR filter. We further employed $\mathcal{H}^{\infty}$ based sampled-data FIR filter design and approximation to derive the optimal digital FIR supporting digital implementation of the proposed control structure. An industry case study was given in detail, where comparisons of the proposed method with existing results were provided in terms of loop shape features and transient behaviors. The TMR analysis showed significant improvement using the proposed method, with more than 15\% tracking TPI capability improvement. Future works based on this methodology include the infinite dimensional $\mathcal{H}^{\infty}$ control for dual stage HDD servo systems and track-seeking control.

\section{Conflict of interest}

The authors declared that they have no conflicts of interest to this work.

\section{References}

[1] Abramovitch D, Franklin G. A brief history of disk drive control. IEEE Control Syst Mag 2002;22(3):28-42.

[2] Chen B, Lee T, Venkataramanan V. Hard disk drive servo systems. Advances in industrial control series, New York, NY: Springer; 2006.

[3] Devasia S, Eleftheriou E, Moheimani R. A survey of control issues in nano-positionin. IEEE Trans Control Syst Technol 2007;15(5):802-23.

[4] Mamun AA, Guo G, Bi C. Hard disk drive: Mechatronics and control. In: Automation and control engineering. Boca Raton, FL: CRC Press; 2007.

[5] Messner W, Ehrlich R. A tutorial on controls for disk drives. In: Proceedings of American control conference. Arlington, Virginia; 2001, p. 408-20.

[6] Yan P, Mckenzie L, Hanson R, Lyle T, Pollock B, Mathur P. Servo systems design for low sample rate disk drive systems. In: Proceedings of the 18th annual ASME conference on information storage and processing systems. Santa Clara, CA; 2008, SCT-B3.

[7] Atsumi T, Messner WC. Estimation method for unobservable settling vibration of head-positioning control in hard disk drives. Mechatronics 2014;23(1):37-45.

[8] Atsumi T, Messner WC. Compensating for ZOH-induced residual vibrations in head-positioning control of hard disk drives. IEEE/ASME Trans Mechatronics 2013;19(1):258-68. 
[9] Chen X, Tomizuka M. A minimum parameter adaptive approach for rejecting multiple narrow-band disturbances with application to hard disk drives. IEEE Trans Control Syst Technol 2012;20(2):408-15.

[10] Kalyanam K, Tsao TC. Two-period repetitive and adaptive control for repeatable and nonrepeatable runout compensation in disk drive track following. IEEE/ASME Trans Mechatronics 2012;17(4):756-66.

[11] Pao L, Franklin GF. Proximate time-optimal control of third-order servomechanisms. IEEE Trans Automat Control 1993;38(4):560-80.

[12] Pao L, Franklin GF. The robustness of a proximate time-optional controller. IEEE Trans Automat Control 1994;39(9):1963-6.

[13] Ohno K, Horowitz R. A variable structure multirate state estimate for seeking control of HDDs. IEEE Trans Control Syst Technol 2005;13(2):233-45.

[14] Dong F, Tomizuka M. An iterative learning control for self-servo writing in hard disk drives using $\mathcal{L}_{1}$ optimal control. In: Proceedings of American control conference. St. Louis, Missouri; 2009, p. 240-5.

[15] Wu S, Tomizuka M. An iterative learning control design for self-servo writing in hard disk drives. In: Proceedings of IFAC world congress. Seoul, Korea; 2008, p. 839-44.

[16] Hong F, Pang CK. Robust vibration control at critical resonant modes using indirect-driven self-sensing actuation in mechatronic systems. ISA Trans 2012;51(2012):834-40.

[17] Huang X, Horowitz R. Robust controller design of a dual-stage disk drive servo system with an instrumented suspension. IEEE Trans Magn 2005;41(8):2406-13.

[18] Young PM, Morris JC, Ho HT. $\mu$-Synthesis control with PI augmentation. In: Proceedings of the American control conference. Minneapolis, MN; 2006, p. $1297-302$.

[19] Atsumi T, Messner WC. Mixed sensitivity problem in sampleddata positioning control systems. ASME J Dyn Syst Meas Control 2011;5(6):1294-309.

[20] Huang X, Lee JI, Ramakrishnan N, Bedillion M, Chu P. Nano-positioning of an electromagnetic scanner with a MEMS capacitive sensor. Mechatronics 2010;20(1):27-34

[21] Sebastian A, Salapaka SM. Design methodologies for robust nanopositioning. IEEE Trans Control Syst Technol 2005;13(6):868-76.

[22] Atsumi T, Messner WC. Optimization of head-positioning control in a hard disk drive using the RBode plot. IEEE Trans Ind Electron 2012;23(1):37-45.
[23] Luo Y, Zhang T, Lee B, Kang C, Chen Y. Fractional-order proportional derivative controller synthesis and implementation for hard disk drive servo system. IEEE Trans Control Syst Technol 2014;22(1):281-9.

[24] Nie J, Conway R, Horowitz R. Optimal $\mathcal{H}^{\infty}$ control for linear periodically time-varying systems in hard disk drives. IEEE/ASME Trans Mechatronics 2013;18(1):212-20.

[25] Li X, Chen S-L, Teo CS, Tan KK. Enhanced sensitivity shaping by databased tuning of disturbance observer with non-binomial filter. ISA Trans 2018;1-9. http://dx.doi.org/10.1016/j.isatra.2018.10.023.

[26] Sanz R, García P, Albertos P. A generalized smith predictor for unstable time-delay SISO systems. ISA Trans 2018;72(2018):197-204.

[27] Şenol B, Demiroğlu U. Frequency frame approach on loop shaping of first order plus time delay systems using fractional order pi controller. ISA Trans 2018;1-9. http://dx.doi.org/10.1016/j.isatra.2018.10.021.

[28] Foias C, Özbay H, Tannenbaum A. Robust control of infinite dimensional systems: Frequency domain methods. Lecture notes in control and information sciences, vol. 209, Berlin: Springer; 1996.

[29] Gumussoy S. Coprime-inner/outer factorization of SISO time-delay systems and FIR structure of their optimal $\mathcal{H}_{\infty}$ controllers. Internat J Robust Nonlinear Control 2012;22(3):981-98.

[30] Yamamoto Y, Anderson BDO, Nagahara M. Optimizing FIR approximation for discrete-time IIR filters. IEEE Signal Process Lett 2003;10(9):273-6.

[31] Yamamoto Y, Nagahara M, Khargonekar PP. Signal reconstruction via $\mathrm{H}-$ infinity sampled-data control theory - beyond the shannon paradigm. IEEE Trans Signal Process 2012;60(2):613-25.

[32] Toker O, Özbay H. $\mathcal{H}^{\infty}$ Optimal and suboptimal controllers for infinite dimensional SISO plants. IEEE Trans Automat Control 1995;40(4):751-5.

[33] Toker O, Özbay H. A Matlab based program for $\mathcal{H}^{\infty}$ optimal/suboptimal controller design. 1996; Publicly available at http://www.ee.bilkent.edu.tr/ ozbay/HINFCON.rar+.

[34] Zhou K, Glover K, Doyle JC. Robust and optimal control. Englewood Cliffs, NJ: Prentice-Hall; 1995.

[35] Meinsma G, Mirkin L, Zhong QC. Control of systems with I/O delay via reduction to a one-block problem. IEEE Trans Automat Control 2002;47(11):1890-5.

[36] Stoecker CA, Misawa EA, Goodner CE. Near time-optimal jerk trajectory for positioning a control object. US Patent 7, 208, 898 B2, USPTO; 2007. 